\title{
ON WEIGHTED EXTENSIONS OF CARLEMAN'S INEQUALITY AND HARDY'S INEQUALITY
}

\author{
TAKUYA HARA AND SIN-EI TAKAHASI
}

\begin{abstract}
Recently K. S. Kedlaya gives a weighted extension of Carleman's inequality under a certain weight condition. However this condition is not essential. In fact we show that the Kedlaya type extension holds without the weight condition by considering a certain weighted extension of Hardy's inequality. Moreover we shall consider the other weighted extension of Carleman's inequality.
\end{abstract}

Mathematics subject classification (2000): 26D15.

Key words and phrases: Carleman's inequality, Hardy's inequality, Young's inequality.

\section{REFERENCES}

[1] H. AlzER, A refinement of Carleman's inequality, J. Approx. Theory 95 (1998), 497-499.

[2] T. CARLEMAN, Sur les fonctions quasi-analytiques, Conferénces faites au cinquième congrès des mathématiciens scandinaves (Helsingfors, 1923), pp. 181-196.

[3] G. H. Hardy, J. E. LitTlewood And G. Pólya, Inequalities, second edition, Cambridge University Press, Cambridge, 1951.

[4] K. S. Kedlaya, A weighted mixed-mean inequality, Amer. Math. Monthly 106 (1999), 355-358. 